\title{
Polar cap magnetic field reversals during solar grand minima: could pores play a role?
}

\author{
Michal Švanda ${ }^{1,2}$, Allan Sacha Brun ${ }^{3}$, Thierry Roudier ${ }^{4}$, and Laurène Jouve $e^{4,3}$ \\ 1 Astronomical Institute (v. v. i.), Czech Academy of Sciences, Fričova 298, 25165 Ondřejov, Czech Republic \\ e-mail: michal@astronomie.cz \\ 2 Astronomical Institute, Charles University in Prague, Faculty of Mathematics and Physics, V Holešovičkách 2, 18000 Prague 8 , \\ Czech Republic \\ ${ }^{3}$ Laboratoire AIM Paris-Saclay, CEA/Irfu Université Paris-Diderot CNRS/INSU, 91191 Gif-sur-Yvette, France \\ ${ }^{4}$ Institut de Recherche en Astrophysique et Planétologie, Université de Toulouse, CNRS, 14 avenue Édouard Belin, \\ 31400 Toulouse, France
}

Received 5 September 2015 / Accepted 21 November 2015

ABSTRACT

\begin{abstract}
We study the magnetic flux carried by pores located outside active regions with sunspots and investigate their possible contribution to the reversal of the global magnetic field of the Sun. We find that they contain a total flux of comparable amplitude to the total magnetic flux contained in polar caps. The pores located at distances of 40-100 Mm from the closest active region systematically have the correct polarity of the magnetic field to contribute to the polar cap reversal. These pores can be found predominantly in bipolar magnetic regions. We propose that during grand minima of solar activity, such a systematic polarity trend, which is akin to a weak magnetic (Babcock-Leighton-like) source term, could still be operating but was missed by the contemporary observers because of the limited resolving power of their telescopes.
\end{abstract}

Key words. dynamo - Sun: magnetic fields - Sun: activity - sunspots

\section{Pores as proxies of Babcock-Leighton source terms}

The Sun is a magnetically active star that exhibits a wide range of dynamical phenomena at its surface and in its atmosphere that are directly related to this activity. The magnetically related activity has been directly observed for more than four centuries, and this record clearly demonstrates that it has been modulated over time variously in its amplitude, frequency of the occurrence of the active phenomena, and the location of their occurrence. In a 11-yr cycle, the global polarity of a solar magnetic field reverses. The reversal of the global magnetic field is prominent in the polar regions - caps - and occurs usually close to the maximum of the cycle (Benevolenskaya 2004). In the current paradigm, fields stemming from the decay of active regions (mostly from their trailing parts) are transported towards the poles by the meridional flow. It is believed that these organised diffuse fields are in effect responsible for the polar field reversals not only at the surface, but possibly deep within the convective envelope leading to a global reversal.

The solar magnetic field is thought to be due to a physical regeneration mechanism termed fluid dynamo, which converts the mechanical energy contained in convective motions into magnetic energy. How such conversion processes operate is subject to debate, but it is generally thought that a largescale shear regenerates the toroidal component of the field, whereas the poloidal component is created via either helical turbulent motions, also known as the $\alpha$-effect, or through the decay of tilted active regions (Babcock 1961; Leighton 1969; Dikpati \& Charbonneau 1999; Brun et al. 2013; Cameron \& Schüssler 2015). This latter process, usually called the
Babcock-Leighton (BL) mechanism, rests entirely on the presence of tilted active regions that continuously emerge on the surface of the Sun during the 11-yr activity cycle. When the magnetic field is strong enough, sunspots may form in these active regions (Parker 1994).

There are, however, periods of very low activity in the recorded activity indices, when the appearance of spots was rather rare according to the observations. These periods (termed "deep" or "grand" minima; Ribes \& Nesme-Ribes 1993; Brooke et al. 2002; Usoskin 2013) are a challenge for the BL dynamo models, as the necessary surface term apparently vanishes. During these large minima observers were still staring at the Sun, however they recorded very few sunspots (e.g. during the Maunder minimum between 1645-1715, see Eddy 1976; Hoyt $\&$ Schatten 1996). Although there was a general conclusion that the solar activity was lower than normal, solar proxies (such as ${ }^{10} \mathrm{Be}$ isotope concentration) suggest that the $11 \mathrm{yr}$ cycle was weak but fairly regular during the Maunder minimum (Beer et al. 1998). Similarly, the geomagnetic activity showed a clear $11 \mathrm{yr}$ variation (Cliver et al. 1998).

When taking the resolving power of the telescopes during the Maunder minimum era (which is estimated to be $2-5^{\prime \prime}$ ), it is possible that the BL term was still operational and that only the organised magnetic field were weak so that the sunspots could not form regularly. In this particular study, we have chosen to focus on a certain class of active regions without sunspots: those containing pores. Pores are white-light features similar to small sunspots. They are believed to be strong concentrations of magnetic field and therefore to potentially possess enough flux for the reversal of the polar field. Therefore, pores might be important agents of the organised magnetic field and were possibly 
invisible to the ancient observers. Compared to sunspots, the pores have a simpler configuration of the magnetic field, which is mostly oriented vertically (Bray \& Loughhead 1964) with strength between 1700 G (Keppens \& Martinez Pillet 1996) and $2600 \mathrm{G}$ (Brants \& Zwaan 1982), depending on the observed spectral line. The pores do not contain a penumbral structure, the majority of them have a diameter of $1500-3500 \mathrm{~km}$, but some are no larger than granules $(700-1500 \mathrm{~km})$. Pores larger than $4500 \mathrm{~km}$ (that corresponds roughly to 5.5") are uncommon.

Unlike sunspots that are mainly confined to a relatively narrow belt $\left( \pm 35^{\circ}\right)$ around the solar equator, the pores were observed up to $75^{\circ}$ (Waldmeier 1955). Also magnetic flux concentrations are spread all the way to solar poles. Shiota et al. (2012) analysed high-resolution Hinode data and found a variety of flux concentrations, some of them having a magnetic flux comparable to the flux of pores. However, no pores in polar regions were detected in the visible-light images.

\section{Purpose of the present work and methodology}

The present work addresses the following question: if we discard the magnetic field in active regions with sunspots, will there still be enough flux to cause the reversal of the polar cap? As said before, we focus on regions containing pores, because they are believed to be weaker than spotted active regions (SARs henceforth) but still contain a significant amount of flux. Moreover, we assume here that such regions with less activity were also present on the Sun during grand minima, as the geomagnetic and heliospheric activity indices tend to indicate. Lites et al. (1998) showed that during the evolution of an active region, the pores start to appear soon after the emergence of the magnetic field into the photosphere. However, the standard scenario of an evolution of the active region (as described by e.g. van Driel-Gesztelyi \& Green 2015) may stop before the formation of proper sunspots. These regions form a low-end tail of the size- and magnetic flux spectrum of active regions (e.g. Hagenaar et al. 2003). Therefore pores appear both near the sunspots and in isolation (Keil et al. 1999), even though it seems that in the space of fundamental parameters, pores and sunspots depict distinct groups (Cho et al. 2015), so one cannot simply say that a pore is a "small sunspot". Nevertheless, it is believed that pores are manifestations of rising flux tubes in the same way as sunspots are.

To study the ability of pores lying outside SARs to maintain the reversal of global polarity of the Sun even during grand minima, we thus have to go through several steps:

- Identify the pores lying outside active regions possessing spots for cycles 23 and 24 where SOHO/MDI and SDO/HMI were and still are available. We consider all active regions containing sunspots and/or pores, however we discard all sunspots and also pores lying close to the cores of spotty active regions. Examples may be seen in Fig. 2, where the ellipse centred at roughly $(1800,2300)$ encircles the region with both sunspots and pores, where the core was discarded, whereas the ellipse centred roughly at $(2150,2500)$ indicates an active region containing only pores. The third remaining active region in this example contains both pores and sunspots only in the core, and all these magnetic features are discarded from the selection.

- For those pores, identify a possible polarity bias. More specifically, we would like to know whether those pores show a polarity dominance in each hemisphere and if this dominant polarity is indeed opposite to the polar cap in the rising phase of the cycle and of the same as the polar cap in the declining phase. Moreover, we give estimates of the flux contained in those detected pores to assess their potential to reverse the polar caps. Indeed, we can only determine their potential for reversal since we should keep in mind that this flux will not be transported entirely to the poles. It has to be noted that in a normal cycle, only a fraction of the total magnetic flux in the typical active regions with sunspots (which is in the order of $10^{23} \mathrm{Mx}$ ) is transported towards the poles (e.g. Sun et al. 2015).

- If a polarity bias has indeed been identified in the previous step, ensure that this is not influenced by the stronger neighbouring SARs. To do so, we wish to determine the "distance of influence" of those regions by determining the possible relationship between the polarity of our detected pores and the polarity of the closest SAR. We will then be able to establish at which distance a SAR may influence the polarity of neighbouring weaker active regions.

- Finally, clearly identify those pores with the right polarity bias in each hemisphere that (if any) are not influenced by neighbouring SARs. Check if they possess the right amount of flux to potentially reverse the polar caps. Then decide on their ability to maintain a Babcock-Leighton surface mechanism even during grand minima episodes.

\section{Data and methods}

\subsection{Data}

We strictly used the archives of measurements obtained by space-borne synoptic experiments. To cover the whole cycle 23 and a progressed part of cycle 24, we combined the datasets from SOHO/MDI ${ }^{1}$ (covering 19 May 1996 to 12 January 2010) and SDO/HMI ${ }^{2}$ (covering 29 March 2010 to 24 August 2014). During the processing, the HMI data were mimicked to be MDI-like (see Appendix A for details), so that the sampling of six hours was used effectively. Higher cadence was only used for testing purposes.

\subsection{Supplementary material}

To assess the total magnetic flux in the polar caps, together with the prevailing magnetic polarity in a given solar hemisphere at a given time, we constructed the magnetic butterfly diagram (the map saturated at \pm 1 Gauss is shown in Fig. 1, top panel). Such a diagram was constructed from the Carrington-rotation synoptic maps ${ }^{3}$. The synoptic maps were averaged over the longitude into a column vector, which forms one column of the resulting magnetic-butterfly map. Since the diagram is computed by means of averaging, the units are in $\mathrm{G}=\mathrm{Mx} \cdot \mathrm{cm}^{-2}$. To get a total net magnetic flux (in Mx), one has to multiply this number by an appropriate surface area.

\footnotetext{
1 We used full-disc intensitygrams downloaded from the online archive at sohowww.nascom.nasa.gov/data/archive/index_ssa.html (there are at most 4 images per day) and corresponding magnetograms stored in the series mdi.fd_m_96m_lev182 at jsoc.stanford.edu

2 Full-disc intensitygrams and magnetograms were taken directly from the series hmi .ic_45s and hmi .m_45s at jsoc.stanford.edu with hourly cadence

3 These maps are available for MDI era at http://soi.stanford. edu/magnetic/index6.html (we used Carrington rotations 19092104), and from series hmi.Synoptic_Ml_720s at jsoc.stanford.edu for HMI era (Carrington rotations 2105-2153).
} 

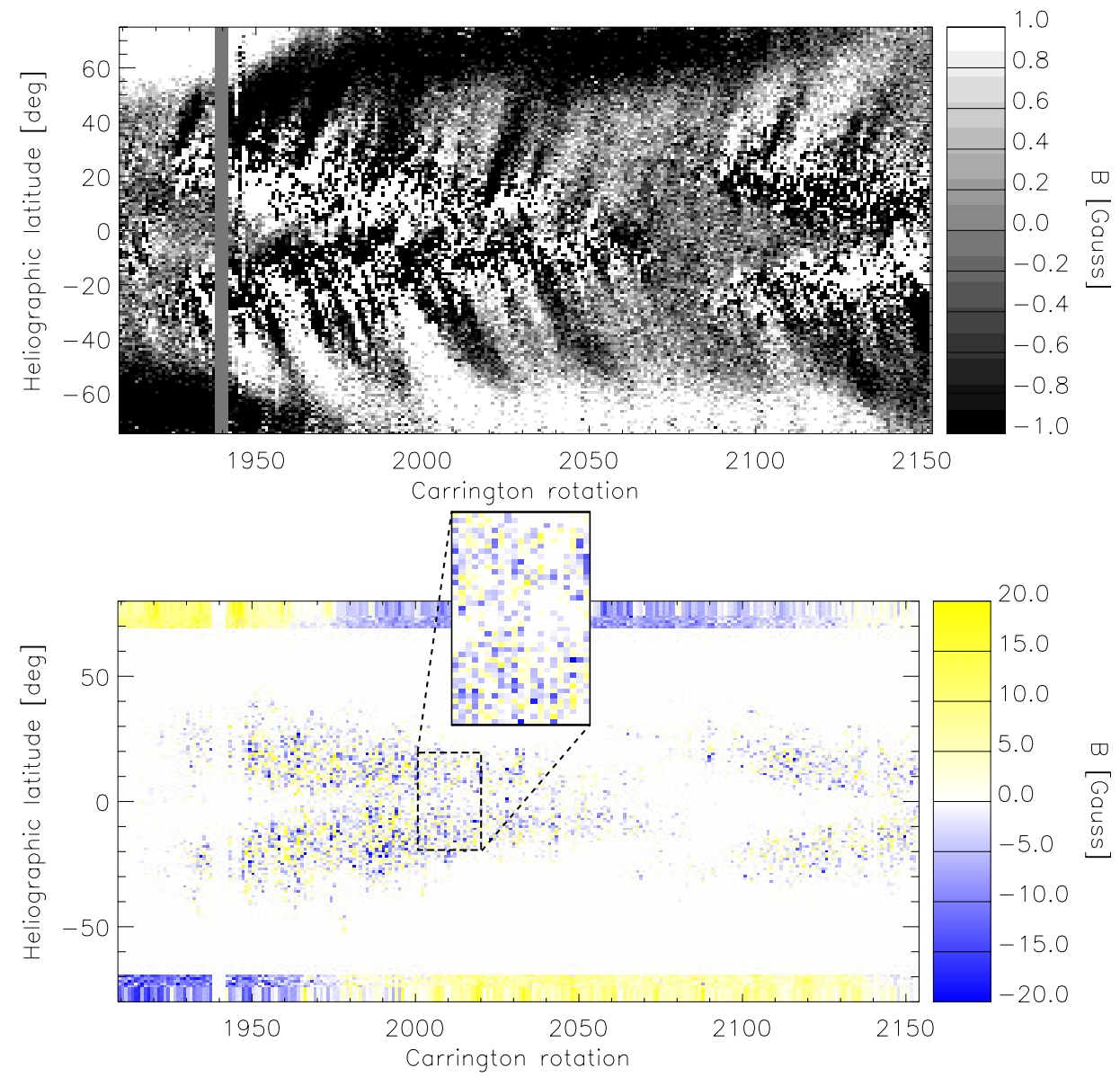

Fig. 1. Top: magnetic butterfly diagram from MDI and HMI synoptic maps shows the prevailing polarity of the magnetic field in polar caps, reversals of the global magnetic field and the flux transported to the polar regions from the relics of the active regions. Bottom: magnetic butterfly diagram of the pores outside active regions. At the top and the bottom the polar cap from magnetic butterfly from above is inserted. The intensity of the average magnetic field in the inserts is boosted by the factor of 5 to make them visible. This figure clearly shows that the pores follow the cycle. The inset in the lower panel demonstrates the mixed polarity in magnification.

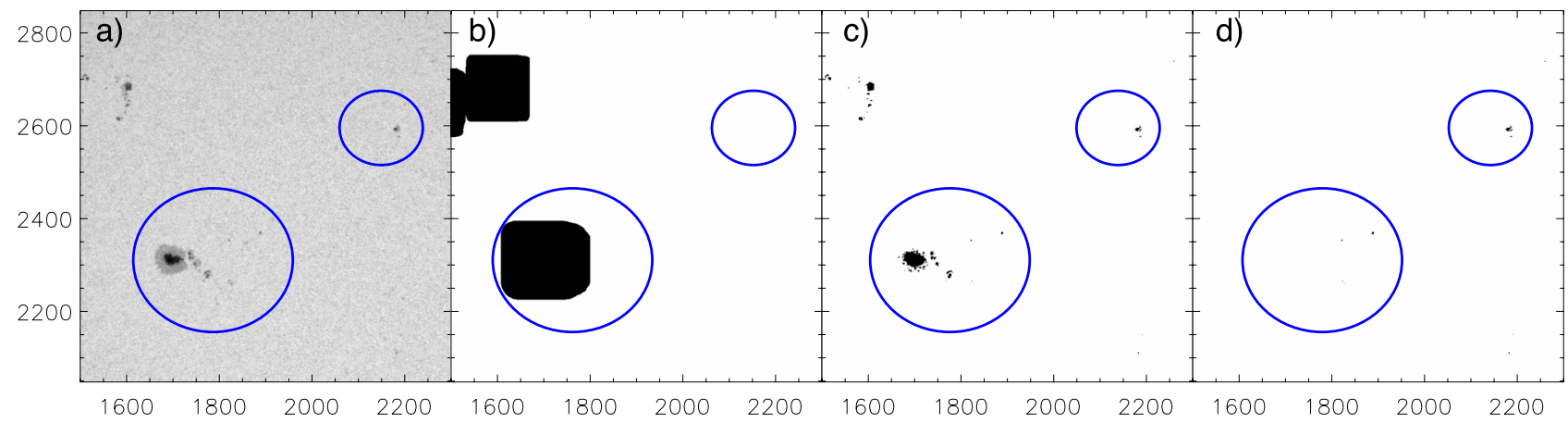

Fig. 2. Steps in the detection of pores outside SARs. a): part of the original HMI intensitygram on 4 Oct. 2014 (axes indicate the pixel positions in the original image). b): mask indicating the positions of active regions. c): mask indicating the detection of all dark features (i.e. spots and pores). d): the final mask segmenting only pores outside SARs. The figures display a section of the full-disc frame captured on 4 October 2014 at 01:00:00 TAI. The ticks on both axes are in pixels, the disc centre is located at coordinates $(2042,2048)$. The pixel size corresponds to $0.5^{\prime \prime}$.

\subsection{Pore detection}

We used full-disc intensitygrams and an automatic IDL procedure to search for the pores. The routine is based on image segmentation and searches for regions of a given size that are significantly darker than the surroundings (by means of thresholding). The search is performed in two steps, differing in the size of the structures that are being looked for. In the first step, rather large areas that correspond to active regions with sunspots are searched for. The mask is obtained by dilation of detected features by 100 pixels, which is later used to exclude the pores in SARs. The small structures, corresponding to the pores, are then searched for in the second step. In this step, artefacts such as dust or bad pixels are also found. These artefacts are removed following the procedure described in Appendix A.

From the set of detected small features only those outside the mask of active regions are kept for further analysis. 
These features are henceforth nicknamed pores. All steps are demonstrated with an example in Fig. 2. The segmentation and labelling is done using standard IDL routines. For each pore, the total area $S$ in squared pixels is computed (when taking the projection effect into account, hence dividing the projected area by a cosine of the heliocentric angle). We further store the mean intensity of the magnetic field in the pore and the positions of the pore in both CCD coordinates (in pixels) and Carrington coordinates (in degrees). Some other useful quantities are also stored, such as the heliocentric angle or effective radius $\left(r_{\mathrm{eff}}=\sqrt{S / \pi}\right)$.

\section{General polarity trends and fluxes for pores detected in cycles 23 and 24}

The appearance of the pores in terms of their positions as a function of time was studied from the combined MDI+HMI data set. All pores fulfilling requirements described in Appendix A were considered. The pores were placed into large maps of various physical quantities describing the pores (average magnetic flux in Gauss, measured area, number of pores in the given coordinate bin) in Carrington coordinates with latitudinal extent \pm 80 degrees and a continuously increasing longitude from 0 (beginning of CR1909) to 88560 degrees (end of CR2153). The map was formed with a binsize of one degree on both axes.

First, we constructed an equivalent to the magnetic butterfly diagram: the line-of-sight magnetic field intensity in pores as a function of time and latitude. Similar to the construction of the reference magnetic butterfly diagram from synoptic maps, we averaged the magnetic flux in pores over longitude separately for each Carrington rotation. The magnetic butterfly diagram of pores outside SARs is plotted in the bottom panel of Fig. 1. From this figure we see that the pores located outside SARs follow the cycle migration for their position, contributing to broadening the activity belt. From the first glance, however, their polarity seems mixed. It is the same situation as with sunspots. They also appear with a mixed polarity (a bipolar sunspot group has both positive and negative parts) and only after the averaging the dominance of one polarity in the given hemisphere appears.

To demonstrate the polarity trends in these pores, we must filter out the random polarity appearance so that only the polarity bias remains, if present. We averaged the magnetic flux in the pores within each of the investigated cycles. The plot is displayed in the upper panel of Fig. 3. When looking at the envelope of the noisy curve, there seems to be a dominance of the positive flux in the northern hemisphere and negative flux in the southern hemisphere in cycle 23, whereas the sign reverses for both hemispheres in cycle 24 .

Using this plot, we may roughly estimate the total magnetic flux in the pores by multiplying the average magnetic field intensity by the corresponding area. The average magnetic field intensity (estimated to be $0.1 \mathrm{G}$ ) was computed using the latitudinal band between $0^{\circ}$ and $60^{\circ}$, which roughly corresponds to an area of $2.7 \times 10^{22} \mathrm{~cm}^{2}$. That would in turn roughly correspond to a total net magnetic flux of $2.7 \times 10^{21} \mathrm{Mx}$. For comparison, the total magnetic flux contained in the polar cap may be similarly estimated from the magnetic butterfly diagram (Fig. 1), where the average magnetic field intensity may be estimated to be $1 \mathrm{G}^{4}$ and the area of the polar cap (everything above $70^{\circ}$ of latitude) as $4 \times 10^{21} \mathrm{~cm}^{2}$. The total magnetic flux in the polar cap is estimated to be $4 \times 10^{21} \mathrm{Mx}$. In the literature, larger total fluxes in polar

\footnotetext{
${ }^{4}$ Such value is consistent with the measurements of the polar fields performed by Wilcox Solar Observatory and published at http:// wso.stanford.edu/gifs/Polar.gif
}

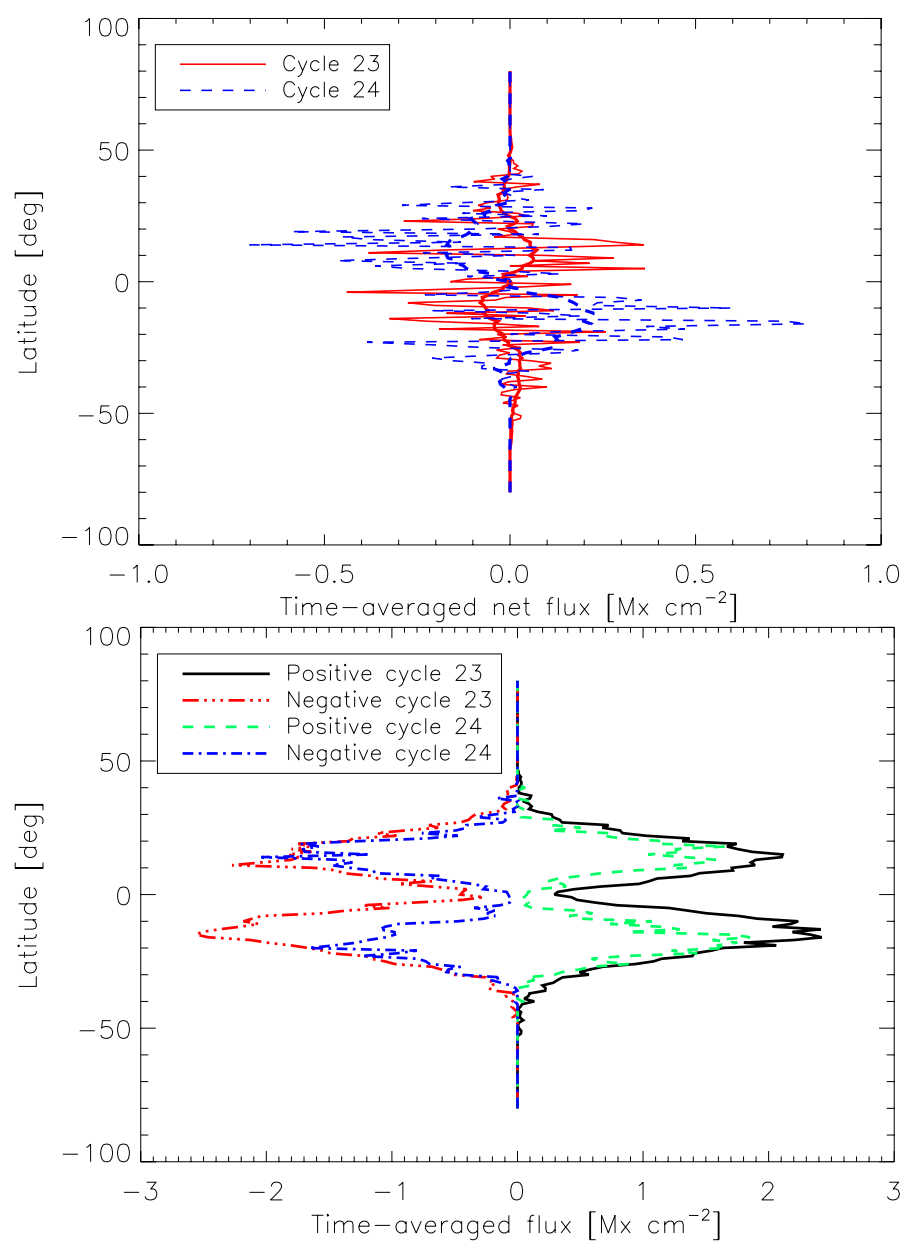

Fig. 3. Upper panel: net magnetic flux in the pores averaged within the cycle over time. In this case the random-like mixed polarity in the pores average out and the secular polarity trends remain. Obviously, in cycle 23 the positive polarity prevails on the northern hemisphere, which reverses in cycle 24 . The thick lines represent the smoothed curves. Bottom panel: magnetic fluxes stored in pores outside SARs averaged over cycles separately for positive and negative polarities.

caps were reported: Babcock (1961) estimated $8 \times 10^{21} \mathrm{Mx}$ (in the minimum between cycles 18 and 19), Sheeley (1966) quoted a varying polar flux between $6 \times 10^{21}$ to $2.1 \times 10^{22} \mathrm{Mx}$ between the years 1905 and 1964 with $1.2 \times 10^{22} \mathrm{Mx}$ being the typical value of the polar flux at the maximum of activity, and Benevolenskaya (2004) reported $1.5 \times 10^{22}$ to $3.7 \times 10^{22} \mathrm{Mx}$ depending on the data used for period of 1996-2003.

A more sophisticated determination of the total polar magnetic flux from various data sets was done by Muñoz-Jaramillo et al. (2012), where the magnetic fluxes in polar caps were found to be around $10^{22} \mathrm{Mx}$. It therefore seems that a value of $10^{22} \mathrm{Mx}$ is a representative number of the total magnetic flux in polar caps in solar cycles in the 20th and 21st centuries, and half this value may be considered representative of a weak cycle like cycle 24 (as a halved amplitude of polar field intensity compared to what cycle 23 suggests). This trend suggests that during very weak cycles, such as during the grand minima, the total polar flux may be even smaller.

Is there a difference in the flux carried by the pores outside SARs in cycles 23 and 24? An additional plot (Fig. 3, the bottom panel) was constructed. It represents averages over cycles 23 and 24 of the butterfly diagram of the pores (Fig. 1 bottom panel) separately for pores with negative and positive magnetic fields. 
In the northern hemisphere, there seems to be a comparable amount of magnetic flux of both polarities in the pores in both cycles. In the south, there is much less magnetic flux in the pores in cycle 24 than in cycle 23 . There has also been fewer sunspots in the south in cycle 24 than there were in cycle 23, at least until 2014 when the southern hemisphere became more active.

One should keep in mind that the analysis of cycle 24 is incomplete because roughly half of this cycle has progressed so far, hence the analysis might be biased. The routines resolved 21403 pores over cycle 23 and 3969 over an incomplete (roughly half) cycle 24 . Taking this possible bias into account, we can still conclude that the pores in cycle 24 have on average a larger line-of-sight magnetic field intensity than pores in cycle 23 .

We do not detect any pores at distances larger than $40^{\circ}$ from the equator. Verma \& Denker (2014) mentioned pores located at slightly higher latitudes $\left(\sim \pm 45^{\circ}\right)$, the difference can be attributed to the higher resolution of the Hinode data they used.

The origin of the polarity trend seen in our pore analysis must be linked to the global organisation of the solar magnetic field through a large scale convective dynamo (Brun et al. 2004; Augustson et al. 2015). Field emergence is believed to be linked to rising subsurface structures whose size, amplitude, twist, and fibril state is still debated and the subject of intense theoretical and observational studies (Fan 2008; Jouve et al. 2013; Nelson et al. 2013; McClintock \& Norton 2013; Schmieder et al. 2014, and references therein). In the next two sections, we look for a spatial and temporal correlation between our selected pores and other magnetic features.

\section{Pores' polarity in cycle 24: influence of neighbouring SARs and relation to the polarity of the polar cap}

Wanting to investigate the feasibility of the pores outside SARs to contribute to the global field reversals, we studied the possible trends in the pores' polarity with regards to the closest SAR and also the polarity of the polar cap of the same hemisphere. To investigate this, we only used the HMI data and dropped some of the requirements of the previous analysis. Mainly, we dropped the limiting requirement on the minimal area of the pore. Now we use all detected pores, including those having an area of only $1 \mathrm{px}^{2}$. For this reason, the total number of pores is much larger. We proceeded in the following way:

1. For each measurement (frames sampled with a $6 \mathrm{~h}$ cadence from the period from 8 April 2010 to 30 May 2014), the SARs were segmented out using a mask. A similar mask to that of Step one in the method outlined in Sect. 3.3 was used to isolate the SARs. The segmentation does not necessarily select complete SARs (both poles) within one mask, it might be that very large open bipolar SARs are detected as two. Then for each pore detected in the same frame using the complete procedure described in Sect. 3.3, the closest (distance measured on the sphere) SAR was identified. The distance is measured and stored. The histogram of distances shows a peak around $30 \mathrm{Mm}$ with a full width at half maximum of around $18 \mathrm{Mm}$ and a long tail towards larger distances. It drops behind $120 \mathrm{Mm}$ and remains almost flat for larger distances.

2. The signs of the flux were compared for the pore and the closest SAR. A "match" tag of the pore is set to 1 when the signs agree and to -1 when they do not. The flux within the SAR is taken as an average over the whole mask.
Usually the total flux in the leading part is larger than in the trailing part, so that the sign of flux within the SAR used in the comparison is that of the leading part. If the SAR is large and bipolar in intensity and open, then it may be detected as two. In this case the sign considered in the comparison is that of the closest pole of such SAR. The methodology of the segmentation is such that the latter case occurs only when the poles of the SAR are at least $60 \mathrm{Mm}$ apart with absolutely no spots or pores in between.

3. Similarly, we compared the polarities of the flux in the pore and the closest polar cap at the given time and set a "match" tag anologically to a procedure described in the previous step.

In the ongoing processing, the pores are binned in the distanceto-the-closest-active-region space (with binsize of $5 \mathrm{Mm}$ ) and in time (binsize of 54 days), and the corresponding tags are averaged. The sizes of the bins were selected so that the resulting maps are sufficiently smooth, because the filling factor of the pores in the maps is low. The binsize in the temporal domain corresponds roughly to two Carrington rotations. For the given distance and time, therefore, if there is a statistically significant match between the polarity of the pore and the polarity of the closest SAR, the averaged-tag value should approach unity. In case there is a systematic mismatch of the two, the averagedtag value should approach the value of -1 . In case there is no obvious rule, the averaged-tag value should be around zero.

In total, more than 118000 individual pores were studied. The top panel of Fig. 4 demonstrates the relation between the pore and the closest SAR. We see two conclusions: (1) there seems to be a significant match between the polarity of the pore and the closest SAR for the pores located between 10 and $40 \mathrm{Mm}$ of the closest AR; and (2) there seems to be a significant mismatch in the polarity of the pore and the closest AR for the pores located between 40 and $140 \mathrm{Mm}$ from the closest AR.

For the comparison between the pore's polarity and the polarity of the corresponding polar cap (see Fig. 4 the bottom panel), the conclusions are different, the trends are not as clear. (1) Pores around $0-40 \mathrm{Mm}$ (most of the pores) from the AR seem to have a mixed polarity compared to the polarity of the corresponding cap. (2) Pores at distances of $40 \mathrm{Mm}$ to $100 \mathrm{Mm}$ (possibly even to distance of $140 \mathrm{Mm}$ ) from the closest AR seem to have the opposite polarity to the cap in the rising phase of the cycle, and the same polarity as the cap in the late rising/plateau phase of the cycle. No conclusions can be made with regards to the declining phase of cycle 24 , since it is still progressing. The trends do not change when both northern and southern hemispheres are investigated separately, including the phase shift of the "reversal" timing in accordance to the delayed polarity reversal in the southern cap in cycle 24 (by about two years). Pores located farther than $150 \mathrm{Mm}$ from the closest SAR do not seem to depict any systematic behaviour.

The pores in the intermediate distances (40-100 Mm, the intermediate pores henceforth) from the closest SAR are especially interesting. These pores are those we were looking for. They have a correct sign to contribute to the reversal of the polar cap. They emerge in the regions of a weaker field, so far enough from the neighbouring SARs. The total magnetic flux contained in the intermediate pores is of the order of $10^{21} \mathrm{Mx}$ (on average in the studied part of cycle 24, each Carrington rotation contains a total flux of $2.3 \times 10^{21} \mathrm{Mx}$ in the intermediate pores in the northern hemisphere and $-0.9 \times 10^{21} \mathrm{Mx}$ in the southern hemisphere). This number is roughly comparable to the total flux in the polar cap estimated to around $4 \times 10^{21} \mathrm{Mx}$ (see Sect. 4). A 

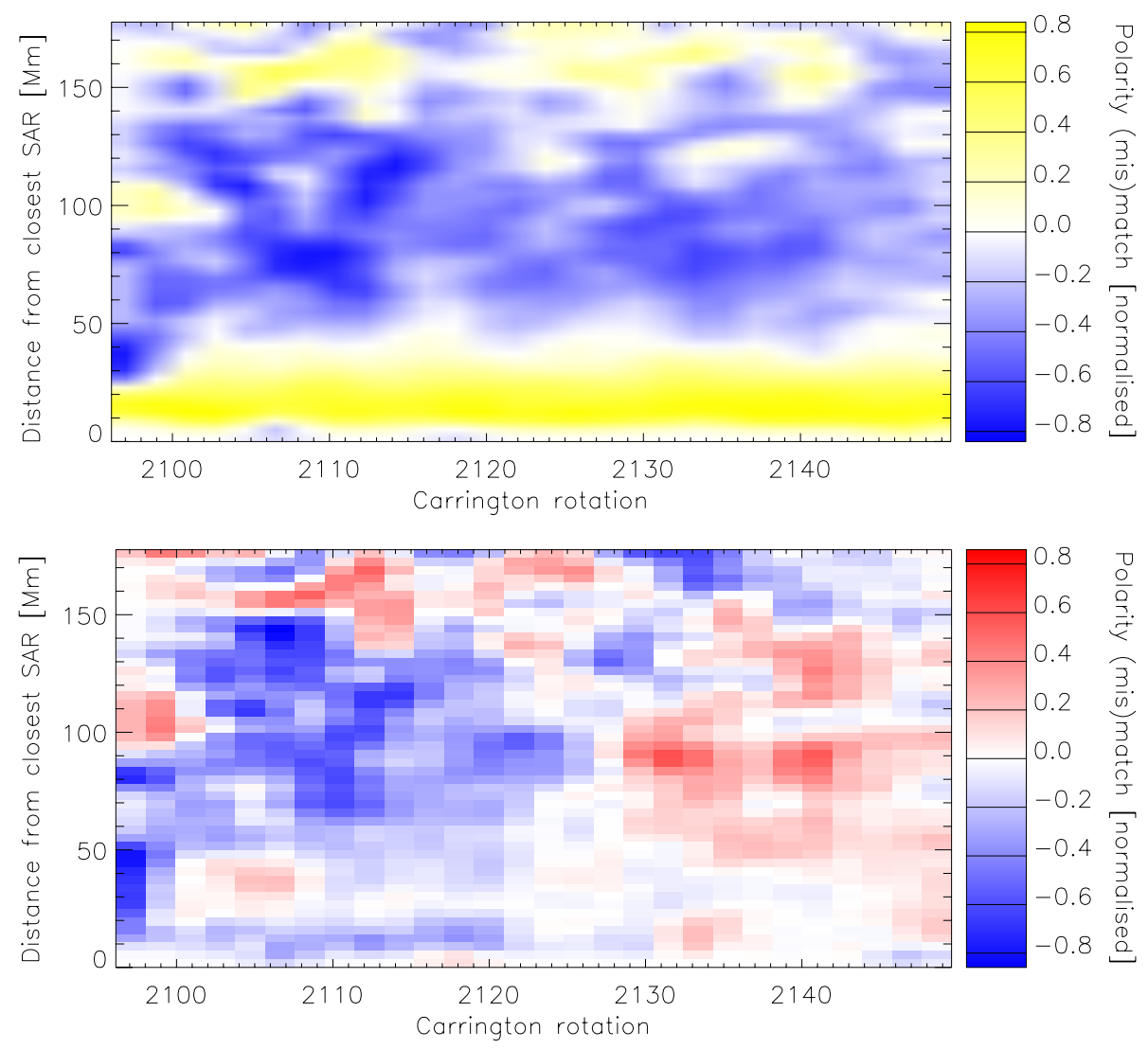

Fig. 4. Upper panel: binned index of match of the flux polarity in pores and their closest SAR as a function of distance from the closest SAR and time. Bottom panel: binned index of match of the flux polarity in pores and the adjacent polar cap as a function of distance from the closest SAR and time. One can see that in the intermediate distances $40-100 \mathrm{Mm}$ the polarity systematically does not match that of the corresponding polar cap in the early rising phase of the solar cycle 24, while they nearly match the maximum of cycle 24. Both panels were smoothed to increase the visibility of the polarity biases. close investigation shows that the pores under discussion reside mostly on the trailing side of the closest SAR.

It is not possible to perform an identical analysis for cycle 23 because it is only covered by MDI observations, which is a lower resolution instrument. The histogram of sizes of the pores shows that the vast majority of detected pores from HMI observations are smaller than $16 \mathrm{px}_{\mathrm{HMI}}^{2}$, hence smaller than $1 \mathrm{px}_{\mathrm{MDI}}^{2}$. The number of pores detected in MDI data over 15 years of MDI observations is only 18683 , while in the HMI data, the same routines detected 118540 pores over four years. This causes figures similar to Fig. 4 constructed for MDI to appear very noisy with many gaps, so they are generally not useful for any serious analysis.

Despite the inability to assess the polarity trends during cycle 23, we may use the findings obtained using HMI observations and focus on intermediate pores. We investigated the flux bias in those pores averaged over the rising and declining phases of cycle 23 and the rising phase of cycle 24 . The splitting was done separately for each hemisphere by using the international smoothed hemispheric sunspot number by fitting a parabola to the points around the suspected minimum and maximum of the given cycle (see Fig. 5).

The time-averaged net magnetic flux in the intermediate pores in the three discussed phases are displayed in Fig. 6. It is evident that the signs of the net fluxes alternate with hemisphere. It is particularly interesting to see that the dominant polarity in the pores during the rising phases are opposite to the polarity of the polar caps and that the polarities then match during the declining phase. This shows the possible ability for those particular pores to reverse the polar magnetic field. We also find that the sign of the net magnetic flux in the mid-latitudes (obtained from the magnetic butterfly diagram by averaging it over the latitudinal bands between 30 and 50 degrees) corresponds to the polarity in those pores. The signs of the prevailing magnetic

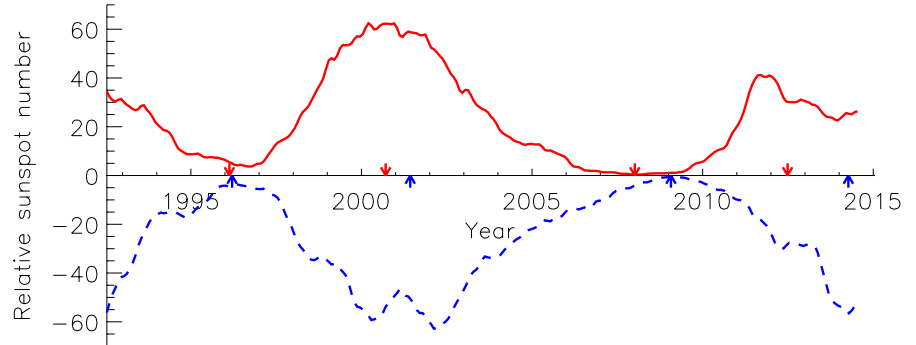

Fig. 5. Sunspot number in both hemispheres in cycles 23 and 24 (the sunspot number on the south was taken as negative for illustration) with the beginning, maximum, and the end of the cycles indicated. (From SILSO data, Royal Observatory of Belgium, Brussels.)

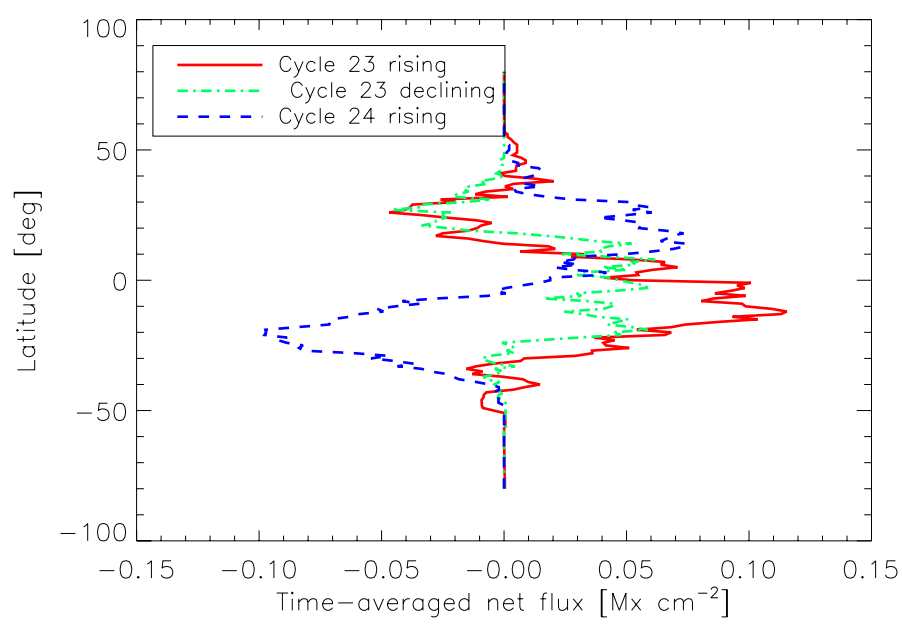

Fig. 6. Net magnetic flux in the pores in intermediate distances averaged over a declining or rising phase of cycles 23 and 24 . 
Table 1. Prevailing signs of the magnetic field of various features in the rising and declining phases of cycle 23 and rising phase of cycle 24 on the northern and southern hemispheres.

\begin{tabular}{lccc}
\hline \hline Feature & $\nearrow 23$ & $\searrow 23$ & $\nearrow 24$ \\
\hline p-spot N & + & + & - \\
pores N & - & - & + \\
mid-lat N & - & $-\nearrow+$ & + \\
cap N & + & - & - \\
\hline p-spot S & - & - & + \\
pores S & + & + & - \\
mid-lat S & + & $+\searrow-$ & - \\
cap S & - & + & + \\
\hline
\end{tabular}

field in various magnetic features (leading spot in the active regions, pores in the intermediate distances from the nearest SAR, mid-latitudinal average magnetic flux, and the polar cap) in the rising and declining phases of the two investigated cycles are summarised in Table 1. Both Fig. 6 and Table 1 thus seem to indicate a possible role for those intermediate pores in the polar field reversal at the maximum of the cycle. Indeed, the flux contained in those pores seems to be carried towards the polar caps until this flux is strong enough to possibly participate in the polar field reversal. The magnetic field in the intermediate pores then continues to be amplified during the declining phase of the cycle before changing sign at the beginning of the rising phase of the next cycle.

\section{Link between pores and bipolar magnetic regions}

The BL surface term relies on the presence of tilted bipolar magnetic regions, which may or may not contain sunspots. By considering only SARs in this study we ignore the presence of bipolar magnetic regions (BMRs) without sunspots and possibly introduce a selection effect in any interpretation of our results. Is it not that the intermediate pores all come from smaller BMRs?

We wrote an additional code to detect BMRs in full-disc magnetograms. We could in principle use the outputs of the HMI Active Region Full-Disk Masks ${ }^{5}$ pipeline and related products; however, they do not cover the MDI era, which is a significant portion of the minimum between cycles 23 and 24 . We have however verified that our pipeline yields comparable results.

Our BMR detection code works as follows. To remove the noise, the magnetograms were first smoothed with a Gaussian window with a full width at half maximum of $15 \mathrm{Mm}$. The code was based on segmentation of the compact patches of negative and positive polarities above the threshold and then pairing them together. We make different choices for the threshold value, where the lowest chosen threshold is 10 Gauss, which is quite low so that we believe we also detect small magnetic regions, and our sample is thus almost complete down to less than the supergranular scales. A pair of the positive and negative patches was marked as a BMR, when they had the shortest distance within the given full-disc magnetogram. The distance metric was modified by penalisation of the distance in the meridional direction, so that we preferred the segmented pairs stretching in the zonal directions.

What is the difference between SAR and BMR? The SARs were obtained by constructing the masks based on the fulldisc intensitygrams, whereas BMRs come from full-disc magnetograms. By construction, each SAR lies within some BMR, and BMR is usually larger by a magnetised rim around SAR.

\footnotetext{
5 http://jsoc.stanford.edu/jsocwiki/ARmaskDataSeries
}
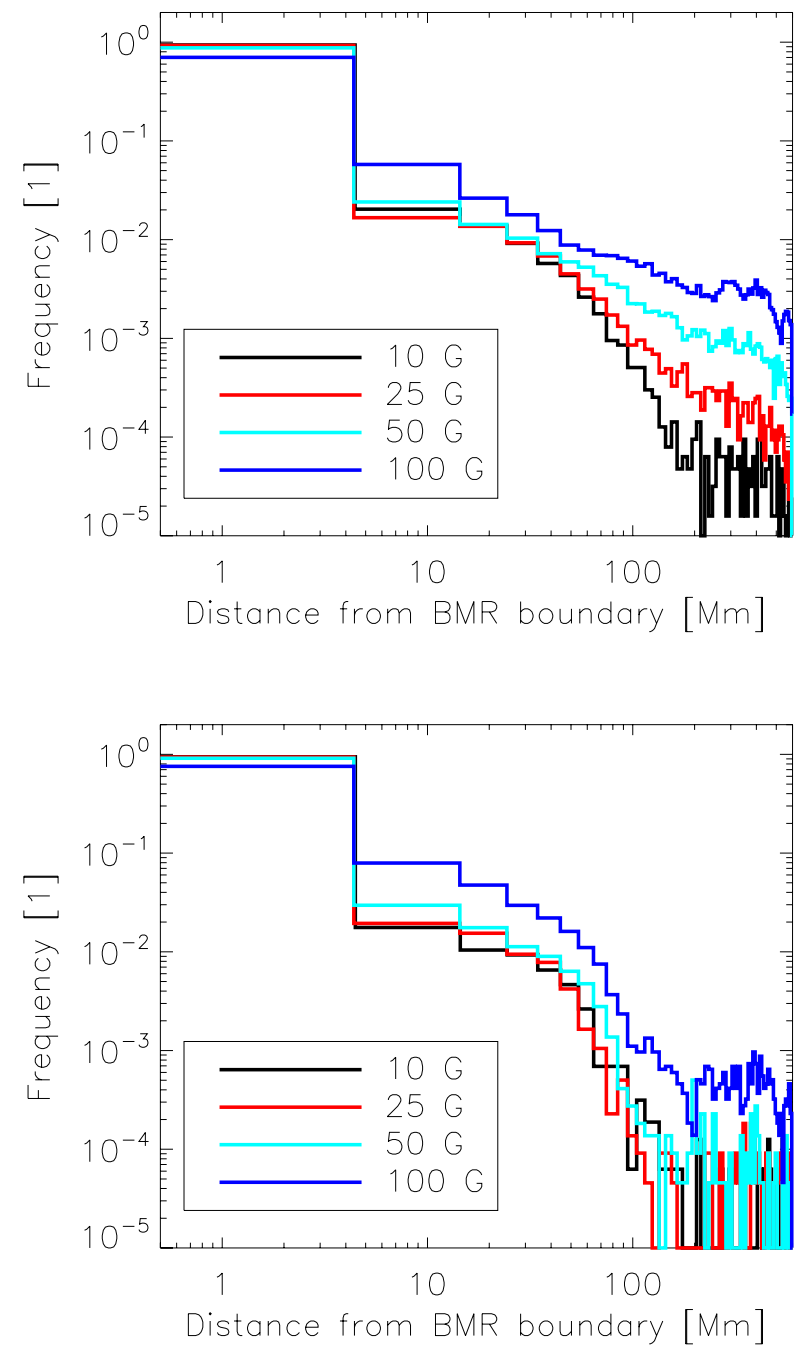

Fig. 7. Histogram of the distances of the pores from the closest boundary of BMR for various thresholds in BMR detection. Upper panel: all pores outside SARs, lower panel: intermediate pores alone. The peak at the distance $0 \mathrm{Mm}$ represents pores located within bipolar magnetic regions detected by the automatic routine. Notice the logarithmic scales.

Additionally, we have a large number of BMRs that do not coalign to any considered SAR, as they do not possess sunspots.

For each pore considered in the previous analysis, we measured its distance to the closest edge of any BMR. In case the pore was located within a BMR this distance was set to zero. The distances were measured for several thresholds in the BMR detection code as described above. The histograms of these distances are displayed in Fig. 7. For the $10 \mathrm{G}$ threshold, 92\% of the pores outside SARs are located within one of the BMRs, $6 \%$ are closer than $30 \mathrm{Mm}$ to the closest BMR, and less than $1 \%$ of the pores outside SARs lie farther than $60 \mathrm{Mm}$ from the closest BMR. The analogical histogram constructed for only the intermediate pores is very similar with an even faster decay towards larger distances (Fig. 7 bottom).

This suggests that pores outside SARs are indeed a proxy for organised bipoles in the photosphere and therefore for a weak BL term. This conclusion seems quite insensitive to the choice of the threshold parameter in the BMR detection code. The decay towards larger distances from BMR is less steep for larger thresholds (when lesser number of BMR is detected), but even 


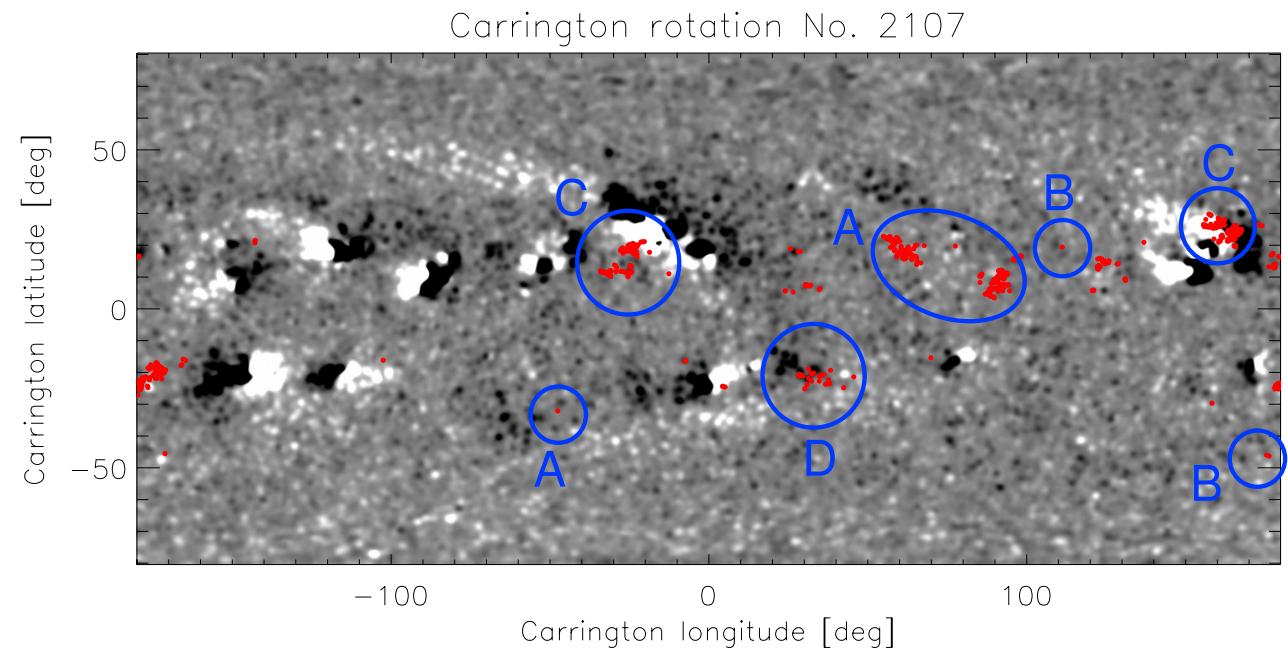

Fig. 8. Example of a synoptic map with various locations of intermediate pores with respect to the closest bipolar active regions. A) The pores can be found in the streams of trailing polarity extending from the dispersed active regions towards poles. B) Pores located far from any obvious patch of organised polarity. C) Pores in or very close to the trailing polarity of bipolar magnetic regions (most of the pores can be found here). D) Pores in or very close to the leading polarity of bipolar magnetic regions.
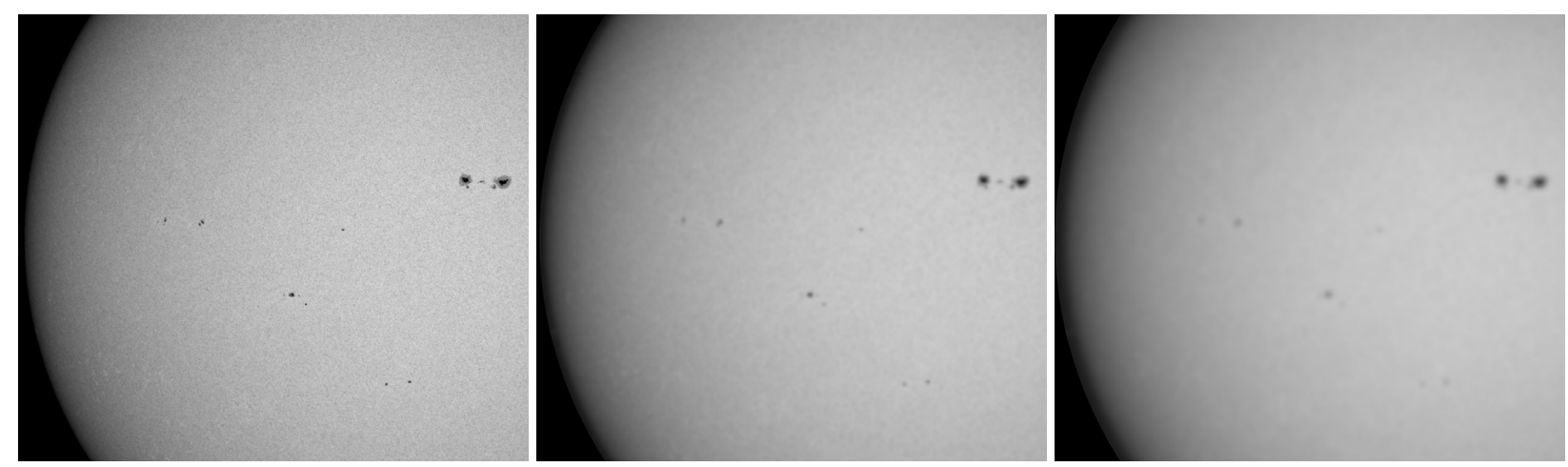

Fig. 9. What ancient observers possibly saw. Left: contemporary white-light image of the Sun captured by HMI from space. Middle: a simulation of what could observers see assuming $2^{\prime \prime}$ seeing and $2^{\prime \prime}$ resolution telescope. Right: the same for $2^{\prime \prime}$ seeing and $5^{\prime \prime}$ telescope. Obviously, the large spots remain perfectly observable, however the small ones are blurred and may be easily missed by the observers, especially when observing in low-contrast conditions (e.g. in a free space).

in this case $57 \%$ of all pores outside SARs $(64 \%$ of intermediate pores) lie within one of the detected BMRs.

A visual inspection suggests that the pores outside SARs may be found either in or next to spotless BMRs or in the polarity streams migrating from the activity belt towards the poles (see Fig. 8). In the consequent Carrington rotation, the pores typically appear at similar locations, creating some sort of persistent nests of pores with a persistence time similar to the lifetime of the adjacent large-scale BMR.

\section{Temporal link between bipolar magnetic regions and active regions with sunspots}

So far we have only considered the spatial distance of the pores from SARs, however it is plausible that the pores we detect are located in the remnants of SARs; i.e., we should also consider a temporal distance. In the previous section we statistically established that a vast majority of the pores under study originate in bipolar magnetic regions. Therefore we investigated whether these BMRs are strictly remnants of SARs or whether they can exist on their own.

Already rough numbers indicate the latter option. For the studied period, the automatic algorithm detected 30418 SARs, whereas a lot more BMRs (depending on the chosen threshold: 26803 for the $100 \mathrm{G}$ threshold, 57247 for $50 \mathrm{G}, 108087$ for $25 \mathrm{G}$, and $207780 \mathrm{BMRs}$ for the $10 \mathrm{G}$ threshold) were detected.
To obtain qualitative results, we investigated the temporal and spatial coalignment of each BMR with all SARs.

For each BMR and also for each SAR, we computed heliographic coordinates and also other descriptive quantities (such as the unsigned total magnetic flux in the BMR). Then we looped over a complete set of BMRs (detected for the $10 \mathrm{G}$ threshold) and searched for a SAR, which was closest to the given BMR's location any time in the past 120 days (more than four solar rotations). The considered distance metric was a distance of gravity centres of the BMR and SARs. By this approach we investigated the possibility of the BMRs being the remnants of SARs.

The results are presented in a form of a histogram of the distances between the BMR and the closest SAR in the past in Fig. 10, which is derived for different classes of BMRs distinguished by the total unsigned flux $\Phi$. Should a vast majority of BMRs be remnants of SARs, the histogram of distances would be strongly peaked around zero. We see that, generally, this is not the case. The conclusions depend on the total magnetic flux in the given BMR. BMRs with a large total unsigned flux, larger than $10^{22} \mathrm{Mx}$, are almost solely remnants of SARs. In our sample, their centres of mass are located less than $70 \mathrm{Mm}$ away from a centre of mass of a previously existing SAR, which existed at this location within the past 120 days. On the other side of the spectrum, BMRs with a small total unsigned magnetic flux, smaller than $10^{20} \mathrm{Mx}$, have a large distribution in distances, basically meaning that they can form on their own and are not linked to classical active regions with sunspots. As seen in Fig. 8 
M. Švanda et al.: Polar cap magnetic field reversals during solar grand minima: could pores play a role?

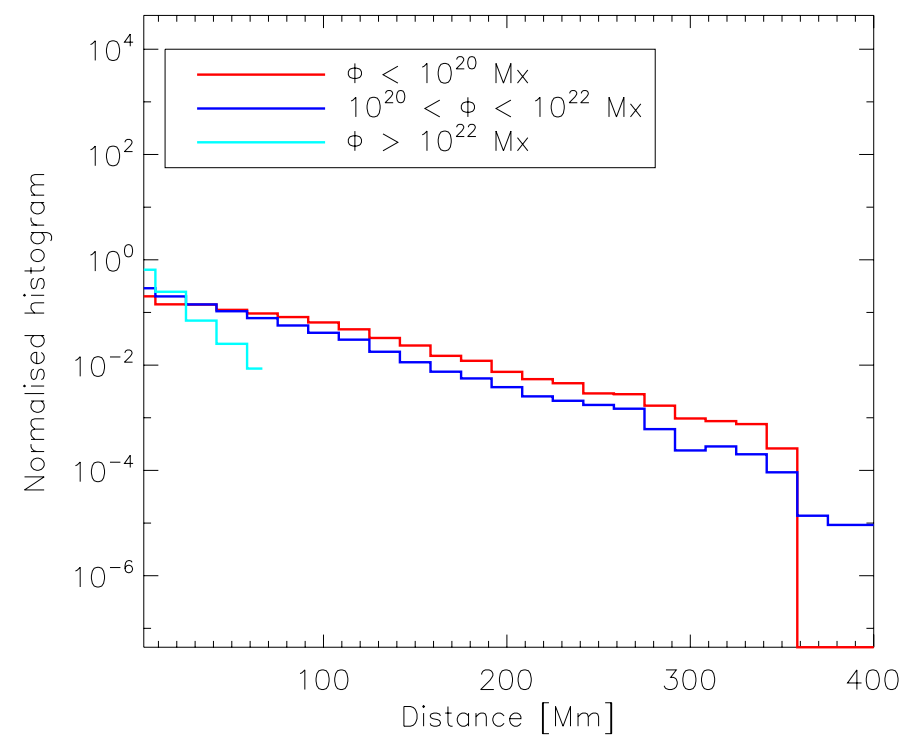

Fig. 10. Histogram of distances between BMRs and past SARs for three classes of BMRs distinguished by the total unsigned magnetic flux.

(feature B), for example, such regions also contain pores. These BMRs may be considered as failed emergence (e.g. Bumba \& Howard 1965a,b) of a raising $\Omega$ loop, in which proper sunspots could not form but in which pores can exist.

\section{Discussion}

Many geomagnetic indices indicate that organised magnetic field must have existed in the solar photosphere even during the Maunder minimum, but there is an obvious lack of positive sunspots observations. The resolving power of instruments of observers of the Maunder minimum era may provide a hint towards understanding the recorded lower activity during this period. Given the expected resolving power of some $2^{\prime \prime}-5^{\prime \prime}$ of the instruments used in the 17 th century, magnetic features such as pores or weak bipolar magnetic regions could have been missed by the observers (for an idea see Fig. 9). This estimate of the resolution limit is supported by a recent thorough investigation through the archival observations of Gustav Spörer, who observed the Sun regularly in the 19th century, hence two centuries after the Maunder minimum. The authors show that his observing limit was around 4" (Diercke et al. 2015).

Indeed when studying the archives, Hoyt \& Schatten described the situation several times in their articles, when a sunspot was observed by some astronomers on a particular day, but not by others on the same day. If we neglect the possibility of an observer's error, it may be that those spots were shortlived, so that they appeared and disappeared during the same day. Similarly, some of these spots could have been small, so that the telescopes of those days resolved those spots for some observers and not for others. Both quantities again resemble the usual properties of solar pores.

A further independent indication that this transition from large to small spots might be operating on the Sun was published by Nagovitsyn et al. (2012). The authors find that as the overall strength of the solar cycle decreases secularly (Penn \& Livingston 2006), there is a transition in the frequency of occurrence of the spots from large ones becoming rare to small ones becoming more common. This conclusion is also supported by a consequent study by Kilcik et al. (2014).
To understand how the lack of sunspot detection is compatible with the continuing existence of a cyclic $11 \mathrm{yr}$ global magnetic field reversal, we have investigated the properties of pores emerging (or forming) at the solar surface during a magnetic cycle, focusing on the ability of such pores, after their decay, to participate in the net magnetic flux needed to reverse the polar caps. Such pores, as explained above, could have been missed due to a lack of resolution power of the instruments used during the Maunder minimum era. We have thus compared the flux carried by the pores outside SARs to the one contained in polar caps, as well as studied any polarity trend of those structures. We indeed found that pores within a distance of 40 to $100 \mathrm{Mm}$ (possibly to $140 \mathrm{Mm}$ ) from the closest SAR do possess the required polarity bias and that most of them are located on the trailing side of the nearest active region. The similarity of the shape of their butterfly diagram and of the butterfly diagram of sunspots also suggest that they could contribute to the operation of the solar dynamo. We also note that in our study no high latitude pores were detected beyond the limit of the activity belt.

We furthermore find that the pores with the correct polarity trend in the rising and declining phase of solar cycle 24 are often found inside weak bipolar magnetic regions (see Fig. 7). Such weak BMRs, in the sense that no sunspots were able to form within them, certainly contribute to the polarity trend found in the intermediate distance pores. Possibly the fragmentation of weak flux tubes (mostly of their trailing leg) forming these weak BMRs after their emergence may be responsible for the occurrence of the pores under study and of their polarity trend. A giant-cell convection may be responsible for such fragmentation so that our finding that the pores with a polarity bias are predominantly located 40-100 Mm (possibly 40-140 Mm) from the closest large active region with sunspots may provide a hint of the length scale of this mode of convection. A "Magnetic range of influence" of emergent flux was investigated by McIntosh et al. (2014) who conclude that the length scale of such a range of influence is between $100 \mathrm{Mm}$ and $200 \mathrm{Mm}$. Also other claims of a detection of cellular features of a similar length scale may be found in the literature.

The link between surface magnetic field and the internal dynamo that produces it is complex, and many scenarios have been proposed over the years to explain this link (Charbonneau 2010; Mackay \& Yeates 2012). One scenario has attracted much attention in the past 20 years, the so-called flux-transport model that relies on the Babcock-Leighton mechanism (Babcock 1961; Leighton 1969). This solar model rests on the observations that much of the magnetic flux is advected by the meridional flow and/or diffused towards the poles. This results first in a cancellation of the polar cap magnetic flux in the rising phase of the cycle and then to the strengthening of the new polarity cap in the declining phase after the global field reversal has occurred (Benevolenskaya 2004; Shiota et al. 2012). Near the equator the trans-equatorial flux cancellation of opposite field polarity helps to renew the global solar magnetic field polarity (Jiang et al. 2015). More specifically, two types of models have been developed over the years that use magnetic flux transport mechanisms as a main ingredient to explain the solar magnetic field and the $11 \mathrm{yr}$ cycle: surface $(\theta, \phi)$ flux models (Wang \& Sheeley 1990; Schrijver et al. 2002; Mackay \& Yeates 2012; Jiang et al. 2014) and meridional $(r, \theta)$ mean field dynamo models (Dikpati \& Charbonneau 1999; Jouve \& Brun 2007; Nandy et al. 2011; Karak et al. 2014). New efforts to couple the two approaches are being undertaken (Miesch \& Dikpati 2014). In this flux transport scenario, the filling factor, size, amplitude, and lifetime distribution of the magnetic features is essential for understanding the 
solar surface magnetism. It would be interesting to adapt our finding of a systematic polarity trend to a pore-like structure to see how efficient it is at reversing weak polar caps.

Of course one must be careful when extrapolating our contemporary study of the pores' distribution and polarity trends back into the Maunder minimum era. We do not attempt here to make a direct comparison but to understand what the poorer resolution of the observations implied back then in terms of missing key surface magnetic features. We conclude that during the Maunder minimum, the Sun could have weak BMRs or small pores that were unobserved but still played a role since we know (thanks to the ${ }^{10} \mathrm{Be}$ content in ice cores, Beer et al. 1998) that the 11 yr cycle dynamo was operating.

Even though the current solar magnetic state is unlikely to be representative of a minimal state of the Sun, we have therefore gained some confidence in our analysis that the Sun is not in a maximum state of activity by noting a) the abnormal length of the transition between cycles 23 and 24; b) the associated large number of spotless days (800); and c) that cycle 24 is much weaker than the last five cycles (Clette \& Lefèvre 2012). Moreover, there have been some claims that the Sun is going to end the current Gleissberg cycle by entering a quieter state with fewer spots (Abreu et al. 2008; Livingston et al. 2012). Since the threshold of $1500 \mathrm{G}$ is necessary for the appearance of dark area (umbra) (Simon \& Weiss 1970; Livingston et al. 2012), a weaker state of activity is likely to lead to a change in the distribution of magnetic features.

\section{Conclusions}

We thus suggest that an emerging magnetic field during the grand minimum did form weak BMRs and associated pores that were undetected. We found that intermediate-distance pores (40-100 Mm from the closest SARs) are good proxies for assessing the polarity trends akin to the weak Babcock-Leighton source term needed to contribute to the polar cap field reversal. The reason these pores and weak BMRs were undetected is probably the lack of resolving power, and the emerging surface magnetic field was not strong enough to form large stable sunspots, unless perhaps for a few cases. We showed in this work that the appearance of pores outside SARs follows the solar cycle in terms, for instance, of the position on the magnetic butterfly diagram.

We also found that the intermediate pores with the correct polarity trend further possess an amount of flux compatible with the flux in polar caps during weak cycles, even it is likely that only a portion of the flux found in pores will be carried poleward. On the other hand, these pores will certainly be surrounded by a larger patch of the magnetic field, which will partly be also carried polewards. We conclude from our pore study that a process akin to a weak Babcock-Leighton magnetic source term could have contributed to the operation of the solar global dynamo during the Maunder Minimum.

Acknowledgements. M.Š. acknowledges the support of the Czech Science Foundation (grant 14-04338S) and of the institute research project RVO:67985815 to Astronomical Institute of Czech Academy of Sciences. A.S.B. acknowledges financial support by CNES Solar Orbiter grant,
CNRS/INSU Programme National Soleil-Terre, ERC STARS2 207430 and SolarPredict 640997 grants and wishes to thank M. DeRosa for useful discussions.

\section{References}

Abreu, J. A., Beer, J., Steinhilber, F., Tobias, S. M., \& Weiss, N. O. 2008, Geophys. Res. Lett., 35, 20109

Augustson, K., Brun, A. S., Miesch, M., \& Toomre, J. 2015, ApJ, 809, 149

Babcock, H. W. 1961, ApJ, 133, 572

Beer, J., Tobias, S., \& Weiss, N. 1998, Sol. Phys., 181, 237

Benevolenskaya, E. E. 2004, A\&A, 428, L5

Brants, J. J., \& Zwaan, C. 1982, Sol. Phys., 80, 251

Bray, R. J., \& Loughhead, R. E. 1964, Sunspots (London: Chapman \& Hall)

Brooke, J., Moss, D., \& Phillips, A. 2002, A\&A, 395, 1013

Brun, A. S., Browning, M. K., Dikpati, M., Hotta, H., \& Strugarek, A. 2013, Space Sci. Rev., 196, 101

Brun, A. S., Miesch, M. S., \& Toomre, J. 2004, ApJ, 614, 1073

Bumba, V., \& Howard, R. 1965a, ApJ, 141, 1492

Bumba, V., \& Howard, R. 1965b, ApJ, 141, 1502

Cameron, R., \& Schüssler, M. 2015, Science, 347, 1333

Charbonneau, P. 2010, Liv. Rev. Sol. Phys., 7, 3

Cho, I.-H., Cho, K.-S., Bong, S.-C., et al. 2015, ApJ, 811, 49

Clette, F., \& Lefèvre, L. 2012, J. Space Weather Space Clim., 2, A6

Cliver, E. W., Boriakoff, V., \& Bounar, K. H. 1998, Geophys. Res. Lett., 25, 897

Diercke, A., Arlt, R., \& Denker, C. 2015, Astron. Nachr., 336, 53

Dikpati, M., \& Charbonneau, P. 1999, ApJ, 518, 508

Eddy, J. A. 1976, Science, 192, 1189

Fan, Y. 2008, ApJ, 676, 680

Hagenaar, H. J., Schrijver, C. J., \& Title, A. M. 2003, ApJ, 584, 1107

Hoyt, D. V., \& Schatten, K. H. 1996, Sol. Phys., 165, 181

Jiang, J., Cameron, R. H., \& Schüssler, M. 2014, ApJ, 791, 5

Jiang, J., Cameron, R. H., \& Schüssler, M. 2015, ApJ, 808, L28

Jouve, L., \& Brun, A. S. 2007, A\&A, 474, 239

Jouve, L., Brun, A. S., \& Aulanier, G. 2013, ApJ, 762, 4

Karak, B. B., Jiang, J., Miesch, M. S., Charbonneau, P., \& Choudhuri, A. R. 2014, Space Sci. Rev., 186, 561

Keil, S. L., Balasubramaniam, K. S., Smaldone, L. A., \& Reger, B. 1999, ApJ, 510,422

Keppens, R., \& Martinez Pillet, V. 1996, A\&A, 316, 229

Kilcik, A., Yurchyshyn, V. B., Ozguc, A., \& Rozelot, J. P. 2014, ApJ, 794, L2

Leighton, R. B. 1969, ApJ, 156, 1

Lites, B. W., Skumanich, A., \& Martinez Pillet, V. 1998, A\&A, 333, 1053

Livingston, W., Penn, M. J., \& Svalgaard, L. 2012, ApJ, 757, L8

Mackay, D., \& Yeates, A. 2012, Liv. Rev. Sol. Phys., 9, 6

McClintock, B. H., \& Norton, A. A. 2013, Sol. Phys., 287, 215

McIntosh, S. W., Wang, X., Leamon, R. J., \& Scherrer, P. H. 2014, ApJ, 784 L32

Miesch, M. S., \& Dikpati, M. 2014, ApJ, 785, L8

Muñoz-Jaramillo, A., Sheeley, N. R., Zhang, J., \& DeLuca, E. E. 2012, ApJ, 753,146

Nagovitsyn, Y. A., Pevtsov, A. A., \& Livingston, W. C. 2012, ApJ, 758, L20

Nandy, D., Muñoz-Jaramillo, A., \& Martens, P. C. H. 2011, Nature, 471, 80

Nelson, N. J., Brown, B. P., Brun, A. S., Miesch, M. S., \& Toomre, J. 2013, ApJ, 762,73

Parker, E. N. 1994, ApJ, 433, 867

Penn, M. J., \& Livingston, W. 2006, ApJ, 649, L45

Ribes, J. C., \& Nesme-Ribes, E. 1993, A\&A, 276, 549

Schmieder, B., Archontis, V., \& Pariat, E. 2014, Space Sci. Rev., 186, 227

Schrijver, C. J., De Rosa, M. L., \& Title, A. M. 2002, ApJ, 577, 1006

Sheeley, Jr., N. R. 1966, ApJ, 144, 723

Shiota, D., Tsuneta, S., Shimojo, M., et al. 2012, ApJ, 753, 157

Simon, G. W., \& Weiss, N. O. 1970, Sol. Phys., 13, 85

Sun, X., Hoeksema, J. T., Liu, Y., \& Zhao, J. 2015, ApJ, 798, 114

Usoskin, I. G. 2013, Living Rev. Sol. Phys., 10, 1

van Driel-Gesztelyi, L., \& Green, L. M. 2015, Living Rev. Sol. Phys., 12, 1

Verma, M., \& Denker, C. 2014, A\&A, 563, A112

Waldmeier, M. 1955, Ergebnisse und Probleme der Sonnenforschung (Lepzig: Geest \& Partig)

Wang, Y.-M., \& Sheeley, Jr., N. R. 1990, ApJ, 365, 372 


\section{Appendix A: Special data treatment}

As we mentioned above, not only are the real solar pores detected by the automatic detection routine, but also artefacts such as the dust or perhaps even bad pixels. These artefacts look exactly like the pores and thus are detected by the algorithm as false positives. That is true especially for MDI in the last years of its operation. They are removed using a simple assumption that their positions on the CCD do not change with time. As a result, all the pores are looped over, and when there is a clustering at the same positions, all these representatives are removed from the set. This correction removes a lot of false positives from the MDI-data-based detections of the pores (in the last years of operation, it was even a vast majority of detections), but has a negligible effect on the HMI-data-based detections of pores.

MDI and HMI are different resolution and sensitivity measurements. Two measures are taken to mitigate this problem:

1. Only features having comparable linear sizes (in $\mathrm{Mm}$ ) are used further for analysis. The MDI pixel is around four times larger than the size of HMI pixel. Two thresholds are thus taken for pores analysed further: a minimum area of the pore must be $1 \mathrm{px}^{2}$ for MDI and $16 \mathrm{px}^{2}$ for HMI, and the maximum area of the considered pore must be below $7.5 \mathrm{px}^{2}$ for MDI and $120 \mathrm{px}^{2}$ for HMI. This way the different resolutions are dealt with.

2. The different sensitivity in line-of-sight magnetic-field measurement is dealt with using a calibration from the overlap period (24 April 2010 to 10 April 2011). Only observations performed by both instruments at exactly the same time (an allowed deviation is $5 \mathrm{~min}$ ) are considered, and pores detected by both instruments were also taken into account. These are strong constraints, since only 202 pores fulfilled them. The considered pores are evenly distributed over the longitudes but are more concentrated in the northern hemisphere. Then the average magnetic field intensity in the detected pores is compared from the two instruments. There is a large correlation between the two (Pearson's correlation coefficient 0.83), and the slope of the linear fit MDI to HMI data is 1.49. (The fit was performed by assuming that both measurements have a random nature.) In the following analysis, therefore, the magnetic fluxes determined from HMI are divided by this number. When the pores of negative and positive polarities are treated separately, smaller correlation coefficients $(\sim 0.3)$ are found, and the slope of the fit is also smaller. Therefore the absolute numbers presented in the paper as the values for the total flux, etc. may be by a factor of the order of unity different based on the calibration slope taken. This factor, however, does not change the conclusions. We note that if the factor were, say, twice smaller than the value used, it would introduce a visible vertical stripe in the magnetic butterfly diagram of pores (Fig. 1) when the transition from the MDI to HMI data occurred.

An additional constraint for dealing with badly assessed detections is that the heliocentric angle must not be larger than 80 degrees. There are often artefacts detected as pores at the limb, which are removed by this constraint.

In total, 49724 individual observations were analysed, 35040 of them from HMI.

\section{Appendix B: Effect of the sampling in time}

We sample the observations with $6 \mathrm{~h}$ sampling. What if we miss some of the pores because of their limited lifetime? What if the pores farther from the equator live a shorter time than those in the equatorial region? In that case we would introduce a systematic error in the pores' distribution at various distances from the equator and hence miss most of the pores at high latitudes, for instance. Currently, we do not observe pores there.

To investigate the effect of the sampling interval, we performed a simple test: the HMI data covering May 2010 and January 2012 were analysed twice using the same routines but with different time steps between the consecutive observations ( $1 \mathrm{~h}$ and $3 \mathrm{~min}$ ). The aim was to search for systematic differences in the distribution of pores in latitudes. This procedure has led to the following results:

- The distribution of pores' locations in latitudes does not change. A shorter time step does not allow detecting pores at high latitudes, so either there are no pores or the detection routine cannot detect them. The latter reason seems probable. Owing to the projection effect, the width of the $2 \mathrm{Mm}$ pore will decrease below the spatial resolution of HMI at already some $45^{\circ}$ from the disc centre.

- The obtained flux maps are highly correlated $(\rho \sim 0.9)$, so the distribution of the flux over the photosphere of the Sun does not depend on the time step.

- The total magnetic flux is 50\% larger for time-step of three minutes than that for the time step of one hour. This would suggest that a lot of flux cancellations occur within one hour.

- The total number of detected pores is expected to be 20times larger from the 3-min-sampled data than from the $1 \mathrm{~h}$ sampled data. (There are 20 non-overlapping $3 \mathrm{~min}$ intervals in one hour.) The test showed that the multiplier is exactly 20 in May 2010. In January 2012 it gets a value of 18.7, so there are fewer pores detected from the 3 min data than expected. This could be explained by the formation of the pores, when the contrast of a forming or decaying pore with respect to the quiet-Sun background is lower than our threshold.

We conclude that the time step only has a weak effect. The nondetection of the pores at higher latitudes is probably due to the projection effect in combination with the spatial resolution of the instruments. 